Apidologie, 1977, 8 (1) 83-88.

\title{
HISTOIRE DU POLLINASTANOL
}

\author{
Zur Geschichte des Pollinastanols
}

Michel DEVYS et Michel BARBIER

Institut de Chimie des Substances Naturelles, C.N.R.S. - 91190 Gif-sur-Yvette

\begin{abstract}
RÉSUMÉ
Une revue est présentée sur le pollinastanol. Cette substance a été isolée pour la première fois des pollens en 1964. Elle a été retrouvée depuis chez divers végétaux et son rôle comme intermédiaire possible dans la biosynthèse du cholestérol a été établi. La structure du déméthyl-4,4' cyloartanol a été proposée et vérifiée récemment par une étude aux rayons- $X$ ainsi que par la synthèse partielle à partir du cycloartanol.
\end{abstract}

\section{SUMMA RY}

THE POLLINASTANOL STORY

A review is presented on pollinastanol. This compound was first isolated from pollen in 1964. Since then, it has been found in several plants and its possible role in the biosynthesis of cholesterol was demonstrated. The structure of 4,4'-desmethyl cycloartanol was proposed for pollinastanol and recently checked by X-ray determination and also by a partial synthesis from cycloartanol.

Comme son nom l'indique, le pollinastanol est un alcool stéroïdique saturé, isolé pour la première fois des pollens. Cette substance, dont la structure est intermédiaire entre celle des alcools triterpéniques comme le cycloarténol, et le cholestérol, offre la particularité d'avoir été le premier déméthyl-4,4' stérol connu ayant un cyclopropane en position 9,19. L'histoire du pollinastanol repose sur une collaboration établie depuis 1957 avec les chercheurs de la Station de Recherches sur l'Abeille et les Insectes Sociaux à Bures-sur-Yvette. 
Cette collaboration a conduit à quelques 45 travaux publiés et nous voudrions choisir cette occasion pour rendre un hommage particulier à Mlle PaIn qui en fut l'instigatrice efficace.

La découverte du pollinastanol est due à un curieux enchaînement de circonstances. Vers 1957, en collaboration avec Mlle PAIN, a commencé une série de recherches sur les sécrétions des glandes mandibulaires des reines et des ouvrières d'abeille. Plusieurs publications s'ensuivirent, dont celles concernant la structure chimique de la substance royale (BARBIER et LEDERER, 1960; Paiv et al., 1962). Environ 2000 reines furent utilisées et une abondante fraction stérolique, biologiquement inactive, fut récupérée et analysée. C'est ainsi que fut découvert le méthylène-24 cholestérol chez les abeilles (BArBier et al., 1959; Barbier et SChindeer, 1959); jusqu'alors, ce stérol n'avait été trouvé que chez des organismes marins, les huîtres par exemple. On a pensé que ce stérol pouvait provenir, en ce qui concerne les abeilles, de leur nourriture habituelle : le pollen; ce qui fut ensuite vérifié par Hügel (Barbier, Hügel et Lederer, 1960; Hügel et al., 1964; Barbier, 1966.)

La découverte du pollinastanol par HüGEL en 1962 a été tout à fait fortuite, à la suite d'une analyse des stérols d'un pollen mixte de composition non déterminée; (Hügel, Barbier, Lederer, 1964). Des eaux-mères de cristallisation déposèrent quelques $300 \mathrm{mg}$ d'un produit nouveau, apparemment différent des habituels stérols. Le caractère accidentel de cette découverte devait causer bien du souci; la recherche de cette substance ne permettrait pas de la retrouver, donnant ainsi à son existence un aspect dogmatique.

Continuant néanmoins son travail sur l'échantillon isolé, HüGEL proposait en 1964 une structure qui devait se révéler être la bonne 10 années plus tard. En effet, les méthodes utilisées alors, ne permettaient pas d'avoir une précision suffisante sur certains points de la stéréochimie de la molécule, laissant planer un doute quant à son rattachement à la famille du cycloarténol; (HüGEL, Barbier, Lederer, 1964; Hügel, 1964).

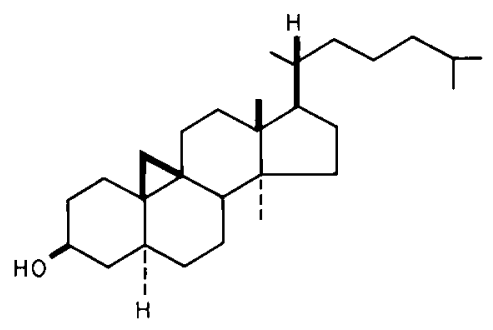

Fig. 1. - Pollinastanol.

Aвв. 1. - Pollinastanol. 
Une recherche systématique du pollinastanol dans des pollens de différentes origines fut entreprise successivement par HüGeL, KREMBEL puis Devys. HüGEL quittait définitivement le laboratoire en 1964, devenant Mme HügeLLhomme, après un stage de perfectionnement dans le service du Professeur Ourisson à Strasbourg. Trois années passèrent et ce n'est qu'en 1967 que le pollinastanol fut retrouvé par Devys dans des pollens de la famille des Compositae : Taraxacum dens leonis et Hypochoeris radicata; (Devys et BARBIER, 1967).

On avait constaté à cette époque que le pollinastanol était toujours accompagné dans les extraits, par le cycloartanol et un stérol en $\mathrm{C}_{27}$, cholestérol ou $\triangle_{7}$-cholesténol. Ceci a permis de supposer l'existence d'une série biosynthétique conduisant du cycloarténol aux stérols en $\mathrm{C}_{27}$ (fig. 2). Cette hypothèse allait mener à deux nouvelles séries de travaux. On pouvait supposer que des végétaux contenant le cycloartanol devaient contenir le pollinastanol, et cette hypothèse devait se montrer fructueuse. Le pollinastanol fut ainsi isolé de la fougère Polypodium vulgare et de la Salsepareille Smilax medica (DEvys et al., 1969). Il est vraisemblable que cette substance est plus répandue dans la nature que ne le laissait supposer sa présence dans les pollens. La possibilité qu'elle joue un rôle d'intermédiaire dans la biosynthèse du cholestérol chez les végétaux fut démontrée par la transformation d'un pollinastanol radioactif en cholestérol par des plants de tabac Nicotiana tabacum; (Devys, Alcarde et Barbier, 1969). Le stérol principal du pollen de la porcelle Hypochoeris radicata (qui contient le pollinastanol) est le cholestérol, ce qui se trouve être en concordance avec l'hypothèse et les résultats obtenus; (DEvys et BARBIER, 1966). La transformation du pollinastanol en cholestérol apportait par ailleurs un argument intéressant en ce qui concerne la stéréochimie de la molécule.

Deux dérivés substitués dans la chaîne latérale, le méthylène-24 et le méthyl-24 pollinastanol étaient ensuite découverts; le premier dans l'algue Chlorella emersonii intoxiquée par le Triparanol (l'accumulation dans cette intoxication souligne le rôle d'intermédiaire) (DOYLE et al., 1972) et dans la peau de banane (KNAPP et al., 1972); les deux substances sont présentes chez le phytoflagellé Astasia longa (Rohmer et Brandt, 1973).

L'existence du pollinastanol et de ses dérivés substitués en position 24, met en évidence des voies possibles de la biosynthèse des stérols $\mathrm{C}_{27}$ (fig. 2) ou $\mathrm{C}_{28}$, procédant par déméthylations en 4 et conservation du cyclopropane, à partir du cycloarténol ou de l'eucalénol. Selon KNapp et al., (1972) le méthylène-24 pollinastanol n'existerait pas à l'état libre dans la peau de banane, mais estérifié par des acides gras.

L'incertitude qui demeurait en ce qui concerne la stéréochimie du pollinastanol, nous a incité à entreprendre une étude par les rayons $X$ (fig. 3 ) et d'autre part une synthèse. L'utilisation des ordinateurs et des méthodes directes 
permettent à présent d'obtenir les structures en quelques semaines, voire en quelques jours si les cristaux sont valables. La preuve que la structure proposée en 1964 était correcte fut rapidement apportée en 1973 (Ducruix et al., 1973; Ducruix et Pascard-Billy, 1976) alors que la synthèse partielle à partir du cycloartanol allait durer deux ans (Bekaert, Devys et Barbier, 1975). L'étude du spectre ${ }^{13} \mathrm{C}-\mathrm{R} M N$ du pollinastanol fut publiée en 1975 (KhUONG-HuU et al.).

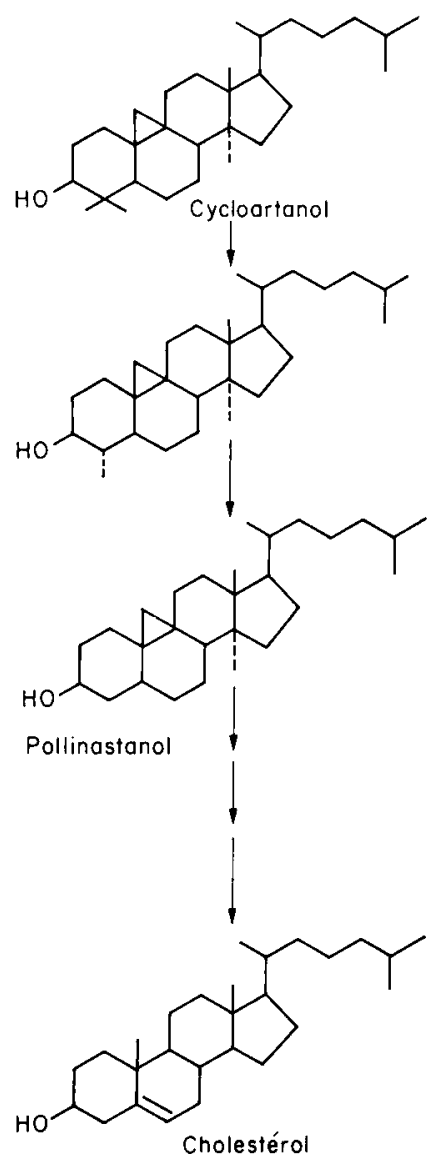

FIG. 2. - Du cycloartanol au cholestérol : situation intermédiaire du pollinastanol.

Aвв. 2. - Vom Cycloartanol zum Cholesterol : Pollinastanol als Zwischenstufe.

Une étude des propriétés physiques (cristaux liquides) de divers esters du pollinastanol a été rapportée (Atallah et Nicholas, 1973). Ces auteurs ont constaté que dans le pollen de Taraxacum dens leonis, le pollinastanol se trouvait principalement sous forme d'esters avec les acides laurique, myris- 
tique, palmitique et stéarique et donc sous forme de cristaux liquides; (pour une revue sur le rôle des cristaux liquides comme constituants des parois cellulaires, voir Bouligand, 1975).

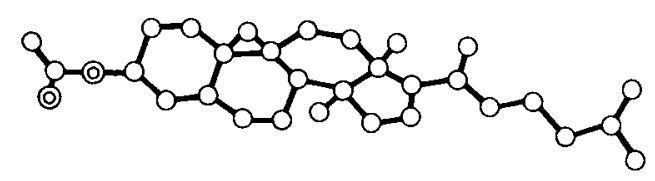

Fig. 3. - Formule de l'acélate de pollinastanol, dessinée par l'ordinateur à la suite de l'étude par les rayons X.

Aвв. 3. - Formel des Pollinastanol-Acetals, vom Computer bei der Röntgenstrukturanalyse gezeichnet.

\section{CONCLUSION}

Neuf années se sont écoulées entre le premier isolement du pollinastanol et la preuve définitive de sa structure. Il est à ce sujet intéressant de noter qu'une étude spécialisée (celle des stérols de l'abeille et des pollens) a conduit à des résultats plus généraux, et ceci, grâce à la persévérance des divers chercheurs qui se sont succédés sur ces problèmes. Le pollinastanol représente en effet une voie possible de la biosynthèse du cholestérol chez les végétaux.

Reçu pour publication en septembre 1976.

Eingegangen im September 1976.

\section{REMERCIEMENTS}

Les plus vifs remerciements sont dus à M. Louveaux, Directeur, à Mlle PaIN, ainsi qu'à tout le personnel de la Station de Recherches sur l'Abeille et les Insectes Sociaux, Bures-surYvette.

Nous remercions les Professeurs E. Lederer et R. Chauvin pour l'intérêt constant qu'ils ont porté à ces travaux.

\section{ZUSAMMENFASSUNG}

\section{ZUR GESCHICHTE DES POLLINASTANOLS}

Es wird über das Pollinastanol berichtet. Diese Substanz wurde 1964 erstmalig aus Pollen isoliert. Seitdem wurde sie in verschiedenen Pflanzen wiedergefunden und ihre Rolle als mögliches Zwischenglied in der Biosynthese des Cholesterols bewiesen. Die Struktur des 4,4' -Desmethyl-Cycloartanols wurde vorgeschlagen und kürzlich durch eine Röntgenstrukturanalyse sowie eine vom Cycloartanol ausgehende Partialsynthese bestätigt. 


\section{RÉFÉRENCES BIBLIOGRAPHIQUES}

Atallah A. M., Nicholas H. J., 1973, Liquid crystalline properties of some pollinastanol fatty acid esters. Molecular Crystals and Liquid Crystals, 24, 213-222.

Barbier M., 1966, Le 24-méthylène cholestérol. Ann. Abeille, 9, 243-249.

Barbier M., Hügel M. F., Lederer E., 1960, Isolement du 24-méthylène cholestérol à partir du pollen de différentes plantes. Bull. Soc. Chim. Biol., 42, 91-97.

Barbier M., Lederer E., 1960, Structure chimique de la Substance Royale de la reine d'abeille. Comptes-rendus Acad. Sc., 250, 4467-4469.

Barbier M., Reichstein T., Schindler O., Lederer E., 1959, Isolation of 24-methylen cholesterol from honeybees Apis mellifica. Nature, 184, 732.

Barbier M., Schlindler O., 1959, Isolierung von 24-Methylen Cholesterin aus Königinnen und Arbeiterinnen der Honigbiene Apis mellifica. Helvetica chimica Acta, 42, 1998-2005.

Bekaert A., Devys M., Barbier M., 1975, Sur la synthèse du pollinastanol à partir du cycloartanol. Helvetica Chimica Acta, 58, 1071-1077.

Bouligand Y., 1975, Les cristaux liquides. Biochimie (éditorial), 57, VII-VII.

Devys M., Alcaide A., Barbier M., 1969, Biosynthesis of cholesterol from pollinastanol in the tobacco, Nicotiana tabacum. Phytochemistry, 8, 1441-1444.

Devys M., Alcaide A., Pinte F., Barbier M., 1969, Pollinastanol dans la fougère Polypodium vulgare et la salsepareille Smilax medica. Comptes-rendus Acad. Sc., D, 269, 2033-2035.

Devys M., Barbier M., 1967, Isolement du pollinastanol et d'une série de $\triangle_{\mathrm{r}}$-stérols des pollens de deux Composées. Bull. Soc. Chim. Biol., 49, 865-871.

Doyle P. Y., Patterson G. W., Dutky S. R., Thomson M. Y., 1972, Triparanol inhibition of sterol biosynthesis in Chlorella emersonii. Phytochemistry, 11, 1951-1960.

Ducruix A., Pascard-Billy C., 1976, Détermination par les rayons-X de la structure moléculaire du pollinastanol. Acta Crystallographica, B 32, 76-81.

Ducruix A., Pascard-Billy C., Devys M., Barbier M., Lederer E., 1973, X-ray crystal and molecular Structure of pollinastanol acetate, a 9, 19-cyclopropane steroid, Chem. Comm., 929-930.

Hécel M. F., 1964, Sur les stérols du pollen. Thèse Doct. Ing. Paris.

HÜGeL M. F., Barbier M., Lederer E., 1964, Sur le pollinastanol, nouveau stérol du pollen. Bull. Soc. Chim., 2012-2013.

Hüget M. F., Vetter W., Audier H., Barbier M., Lederer E., 1964, Analyse des stérols du pollen par spectrométrie de masse. Phytochemistry, 3, 7-16.

Khuong-Hud F., Sangare M., Chari V. M., Bekaert A., Devys M., Barbier M., Lukacs G., 1975. - Carbon-13 nuclear magnetic resonance spectral analysis of cycloartanol and related compounds. Tetrahedron Letters, 1787-1790.

Knapp F. F., Philipps D. O., Goad L. J., Goodwin T. W., 1972, Isolation of $14 \alpha$-methyl - $9 \beta-19$ - Cyclo $-5 \alpha$ - ergost - 24. (28)- en $-3 \beta$ ol from Musa sapientum. Phytochemistry, $11,3497-3500$.

Pain J., Barbier M., Bogdanovsky D., Lederer E., 1962, Chemistry and biological activity of the secretions of queen and worker honeybees. Comp. Biochem. Physiol., 6, 233-24l.

Rohmer M., Brandt D. R., 1973, Les stérols et leurs précurseurs chez Astasia longa. European J. Biochem., 36, 446-454. 